\title{
NUEVOS CASOS DE AMANITISMO EN CHILE
}

\section{New cases of amanitism in Chile}

\author{
Waldo Lazo \\ Departamento de Ciencias Ecológicas \\ Facultad de Ciencias - Universidad de Chilc
}

Palal)ras clave: Amarita phalloides. micetismo

Key words: Amanita phalloides, micclism

\section{RESUMEN}

Nueve personas padecieron de un serero micerismor en jumior de 1999 por comer havidiomas de Amanita phalloides (liaill. ex lir.) Secr: recolectados en Linares (I'll Region). Dos de los pacientes fallecieron. Se hace referencia rambien a algunos de los hongos venenosos más commenes èn chrile.

Los micclismos morlales son hechos. más bicn csporádicos en Chile, a juzgar por las comunicaciones realizadas durante los últimos 65 años (Barriga. 1935; Mardones el al., 1935: Mcza. I935: Vicuña el al.. 194t: Cabrera. 19+6: Beln \& Jerardino. 1967: Lazo. 1982: Valenzuela er al., 1992: Sicrralla elal., 1994).

Las especies cuyos basidiomas, al ser ingeridos. provocaron los curenenamientos son: Lepiota locaniensis Espinosa. Amanita toxica (Lazo) Garrido el Bresinshy. Almanitophalloides (Vaill. ex Fr:)Secr.

L. Locamiensis al pareccr scria ya una especie extinta (hace ya más de 50 años que no ha sido vista ni recoleclada en los lugares donde se desarrollabal). Amamia rovica ha sido cnconurada descle la V liasla la X Región desde 1967 hasia ahora. En 1967. a csla especic se la idenlilicó como Amanita gémmata. cn 1982 como Amanita gemmata var. toxica (Lazo 1982) y en 1985 como Amomita toxica. (Garriclo \& Bresinsky. 1985) Este último cs su nombre válido. El micelismo (a veces mortal).calusado por csla especie. cs icléntico o casi iclénlico. al causado por $A$. phalloides, A.verna o A. virosa, annque en su basidioma no se lia enconlaado ninguna amanitina. sino un compucsto semejante a la ouabaina (Sacz el al., 1981). Valenzuela et al. (1.992). commicaron la ocurrencia de micclismos mortales cn la IX Región décidos a Ia ingestion de basidiomas de A. phalloides y' Sicrrallia el al., (1994). proporcionaron abundante información sobre micctismos ocasionados por ćsta y’ otras especies en lá IX Región.

\section{SUMARY}

l/fer eating Amanita phalloides (Faill. ex Fi:) Secr: basidiomara collecled in linares (IFIl Región), o persons suffered a severe mycetisin in june 1999. Tho of the'll died, the ollier seven recovered after medical treament. Bessides some of the poisornous fungi most commonly found in Chile are herein memioned.

El 13 de junio de 1999, un grupo de personas ingirió unas llamativas setas de color verde limón recolectadas en Jals ccrcanias de la ciudad de Linalles (VIl Región). Den(ro de lis doce horas siguientes los comensiles presentaron sintomas de gastroenteritis agnda que se remitieron tras un tratamiento sintomático simple. Horas después "la indigestión" rolvió y el 17 de junio, al empeorar nolablemente algunos de ellos, fiteron internados en la U.T.I. de una clínica. solicitándose a un micólogo la identificación cle las setas ingeridas cuatro clias antes. Afortumadamente muchas de ellas no alcanzaron a ser cocinadas y algunos ejemplares aún se mantenúan on el refrigcrador. Estas fueron identificadas como Amanita phalloides. En realiclad. correspondian a los más calacleristicos basidiomas de csla especic que este autor haya risto. A partir de ese día los pacientes recibieron el tralamiento médico indicado para esla patología. Sicle de ellos se recuperaron y dos lállecieroll.

Las caraclerist icas de ,4.phalloides (Fig. I superior) son: Pilco de $6-15 \mathrm{~mm}$. de diámetro. verde amarillento (verde limón) a gris oliváceo. con fibrillas radiales de color oliváceo (hay una forma con la cara supcrior del pílco de color blanco): ovoide a subgloboso cuando nuevo, aplanado convero al envejecer: superficie sattinada cuando seca, líbrica si cstá mojada. Lamelas libres, blancas, frecuentemente amaril lo verclosas en el margen, clesiguales. Estípite de 8-20 × $1-2 \mathrm{~cm}$.. blanco, aumenta su grosor hacia la balsc donde forma un bulbo ovoicle. Volva saciforme basal os- 

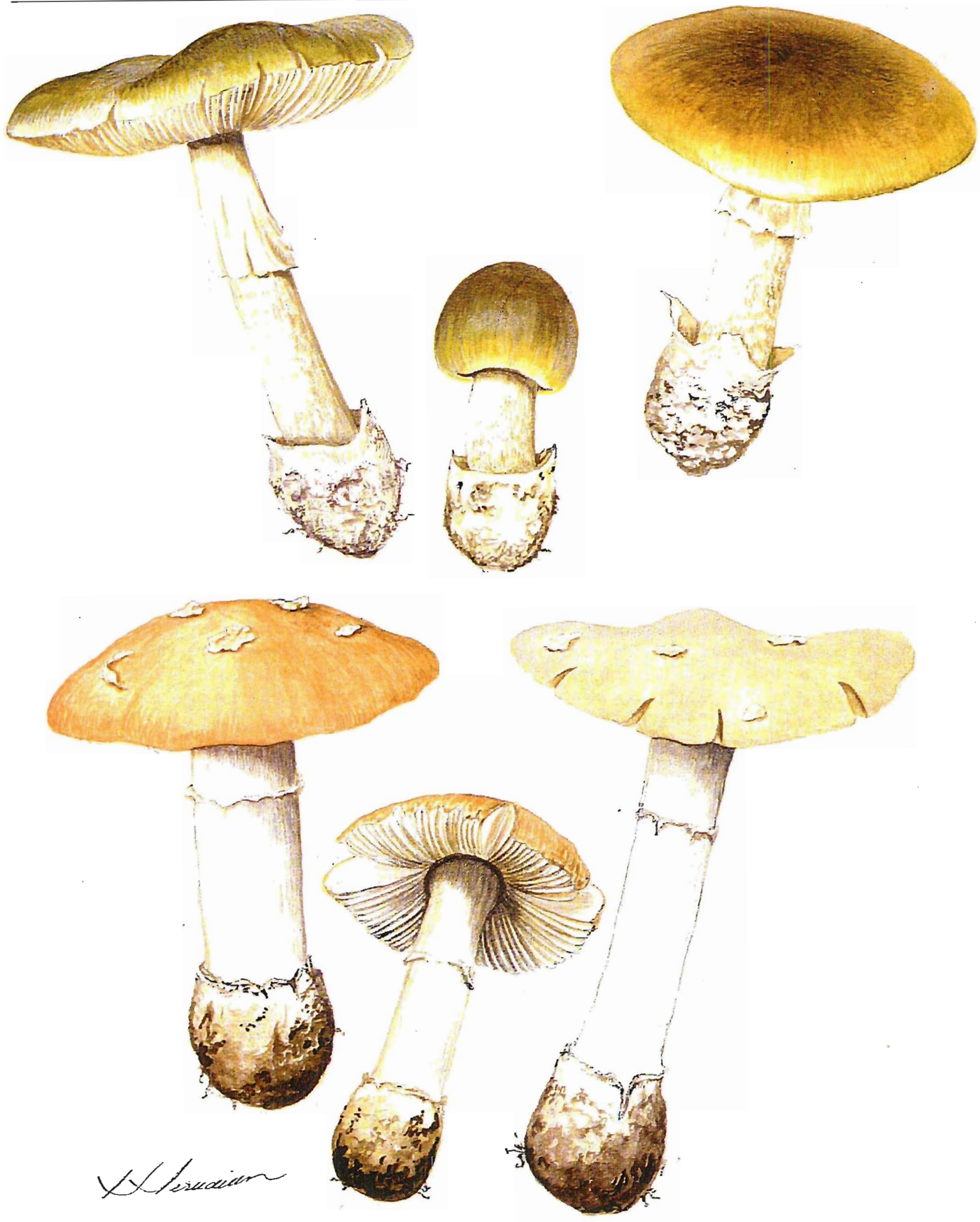

Fig.1 Amanita phalloities (superior) y Amanita toxica (inferior)

(Acurrelas de. E.J. Bernain Desmaras) 
tentosa (característica muy imporlante para la identificación). Anillo blanco, apical, persistente. Contextura carnosa, suculenta, blanca. Sabor clulzón. Olor a miel o a rosa marchita cuando nucro, putrescente en Ja madurez. Esporas de 8-1.3 $\times$.7-9 $\mu m$, hialinas, amiloides, subglobosas, lisas. Basidios de 32-4() $x+10 \mu$ m. Tetrasporados, claviformes. Pilcipelis filamentoso.

La reacción de Wieland delata la presencia de amatoxinas en los basicliomas.

II abitat: en el suelo, en bosques de Pimus racliara, Quercus spp., Nollhofagus spp. y' otros. Distribución: amplia (Europa. América, VII y' IX Región en Chile).

Las Características cle $A$. toxica (Fig. I, inferior) son: Píleo de 55-77 mm de diámelro, blanquizco, con la zona central cle color parduzco suave (beige) o, a veces, con loda la cara superior del pílco parduzca amarillenta, plano y algo deprimiclo en la maclurez, cscamas cn la zonia central. Lamelas libres, blanquizcils, desiguales, Eslípite de 60-130 x 10-20 mm. blanquizco, cscuamuloso. Anillo blanco, frígil. Volva blancá. Esporacla blancai, Esporas de 8-」1. 7-9) pum, hialinas, globoides, lisas, apiculadias, inamiloides. Basidios de $4(0-50 \times 8-10 \mu \mathrm{mm}$. Hiallinos, letrasponados. Hay plemrocisticlios y' qucilocisticlios. "Trama no bilatcral, sino entremezclada cn los cicmplares adululos. Lil presencia de fíbulas en la base de los basidios e hifas de la pileipellis permilen separarla de $\boldsymbol{A}$. gemmut $($ Lazo 1982), caracleres que permitieron postularla como una nueva especie (Garri(lo \& Bresinkt; 1985).

II abitat: en el suelo, dentro de bosques cle Pinus radiata, Eucalyptus spp. u otros árboles. Distribución: ha sido recoleclada clesde la V Región (Peñuelas) hasıa la X Región.

De las 28 especies fúngicas tóxicas ciladas por Garrido(1985), debería señalarse especialmente: Agaricus .canthodermus, Amanita muscaria, Hebeloma crustuliniforme, Hy groclye conica, Lepiota cristata, Panacolus retirugis, Panacolus sphinctrimus, Paxillus involutus, Psiloscybe semilanceata, P. zapotecorum, Rhodocybe mundula, Tricholoma pessundatum. Mención aparte merece G. rom!ny/ra antarctica, cuyos ascomas provocaron una ostenlosa patologia digestiva a 2 muchachos que comieron sus ascomas crudos durante una excursión campestre en 1966. Al parecer, a veces, alunque estén cocidos, ćslos pueden ser indigeslos.

El cuaclio I resume los tipos de micetismos ocurridos en Chile durante los últimos 65 años, en muchos de cllos, este aulor turo conocimiento directo, ver aclemás Sicrralla clal., 199t.

\section{REFERENCTAS}

Barriga, M. (1935). Intovicationes por hongos. Bol. Soc. Med. Chil: $63: 580-581$

Behn, I: \& Jerardino, M. (1967). Intoxicaciones con Amanita gemmatn (Fir) Gillet, Nutr. Bromatol. Toxicol. 6:15t

Cabrera, R. (1946). Ilongo chileno venchoso. Agr. Tes. Chile $6: 7 \AA$

Concha, J.; Ginrrido, N. \& Mayer, I1. (1979) Amanitu gemmata. Identilicación, distribución y posible prevención de su loxicidand. Arelı. Biol. Med. E.sp. 12:506

Concha, J.; Herrera, R.; Mayer; H.; Síc\%, J. (1979). l'urilicición parcial de compuestos con aclividad biological presentes en Amaniar gemmata. Archiv: Biol. Med. Exp. 12:506

Espinosa, M. (1936). Un hongo nuevo chileno. Bol. Mlus. Nac. Chile $75: 81-89$

Garrido, N. (1985). Index Agaricalium Chilensis. Bibliolhecil Mycologici 29. I.Cranmer: Vádù.

Garrilo, N. \& Bresinsky, A. (1985). Amanita mersmuelleri. (Agaricales) eine neve Artitus Nolholigus-Waldem Chiles. 13ot. Jahrb. Syst. 1()7:521-541)

Lazo, W'. (1982). Ilongos venenosos en Chile. Bol. Inst. Salud Prublical (Chile) 23:122-126
Marilones R. \& Vilal. (1935). Intoricaciones por hongos. Bol. Soc. Med. Chile. 63:588-589

Mayer, II.; Sace, J.; IIerrera, R.; Concha, J. (1980). Intoxicación aguala por hongos. Rev. Méd. Chile. 108:671-672

Ne\%a, O. (1935). Necesidad de mediclas samitarias para prevenir posibles intoricaciones por hongos alimenticios. Casos Clinicos. |3ol. Soc. MĖl. Chile. 63:581

Pacciomi, G. (19S2). Guia de IIongos. El. Grijalbo. Barcelona.

Síc\%, J.C.; Mayer, H1.; Cifuentes, I.; Concha, J. (1981). A oulabaine like compound present in Amamita gemmatı (Fr.) Gillet. IRCS Merlical Science, 9:297-298

Sicralta A.; Jeria M.; Figucroa G.; P'into J.; Araya J.; Sin Juan J.; Grinbergs J.; Valenzuela, E. (1994). Intoxicación por callampas lenenosas en la Lل Región. Rol de almanita gemmartr. Rev. Mel. Chile. 122:795-802

Valenzucla li.; Moreno G. \& Jeria M. (1992). Amanira phalloides en hosques de Pinus radiata de la IX región de Chile:'laxonomiat, toxinas, métodos de detección, intoxicación lialoidiant. Bol. Micol. 7:17-21

Vicuña R.; Philippi f. \& Melendey O. (1944). Inloxicaciones por hongos. Rev. Med. Chile. 72:986-95 
Cuadro 1.-

HONGOS Y MICETISMOS

\begin{tabular}{|c|c|c|c|c|c|}
\hline SINDROME & VENENO & ESPECIE & INCUBACION & TRASTORNOS PRINCIPALES & DURACION \\
\hline Faloidínico & $\begin{array}{c}\text { ciclopéptidos } \\
\qquad ?\end{array}$ & $\begin{array}{l}\text { Amanita phalloides } \\
\text { Amanita toxica }\end{array}$ & $8-36$ horas & $\begin{array}{l}\text { Daño hepático y renal, } \\
\text { vómitos, diarrea, cólicos. }\end{array}$ & $\begin{array}{l}10-20 \text { dias, } \\
\text { muchas veces letal }\end{array}$ \\
\hline Parafaloidinico & ¿ciclopéptidos? & Lepiota locaniensis & $5-15$ horas & $\begin{array}{l}\text { Daño hepático y renal, } \\
\text { vómitos,diarrea y cólicos }\end{array}$ & $\begin{array}{r}\text { Datos imprecisos, } \\
\text { muchas veces letal }\end{array}$ \\
\hline Giromitrico & monometilhidracina & Gyronytra suntarctica & $2-4$ horas & $\begin{array}{l}\text { Sintonatología gastrointestinal } \\
\text { y sistema nervioso }\end{array}$ & $\begin{array}{l}5-6 \text { dias, a } \\
\text { veces letal }\end{array}$ \\
\hline Alucinógeno & psilocibina, psilocina & $\begin{array}{l}\text { Psilocybe :apotecormm } \\
\text { Psilocybe semilanceata } \\
\text { Gymnopilus purpuratus } \\
\text { Mycena pura }\end{array}$ & 0,5 - 4 horas & Alucinaciones y delirios & $1-3$ dias \\
\hline Muscarinico & 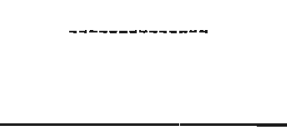 & $\begin{array}{l}\text { Clitocybe suaveolens } \\
\text { Clitocybe spp. } \\
\text { Inocype spp. }\end{array}$ & $0,5-2$ horas & $\begin{array}{l}\text { Bradicardia,lipotensión } \\
\text { arterial, miosis, diarrea }\end{array}$ & $1-3$ dias \\
\hline Paxílico & & Paxillus involntus & $1-8$ horas & Diarreas, vómitos, trastomos cardiocirculatorios & $2-4$ dias, a veces letal \\
\hline Botulinico & & $\begin{array}{l}\text { Basidiomas clescom- } \\
\text { puestos o alterados }\end{array}$ & $1-4$ horas & Digestivos y del sistema nervioso & $\begin{array}{l}3-5 \text { dias, a } \\
\text { veces letal }\end{array}$ \\
\hline Coprinico & coprina & Coprinus spp. & $\begin{array}{l}\text { Lunediatamente } \\
\text { espués de beber alcohol }\end{array}$ & Sistema digestivo y nervioso & $1-2$ dias \\
\hline $\begin{array}{c}\text { Envenenamiento } \\
\text { iboténico }\end{array}$ & $\begin{array}{l}\text { ácido iboténico, } \\
\text { muscinnol }\end{array}$ & Amanita muscaria & 0.5 a 2 - hrss. & Alucinaciones & $2-4$ hrs. \\
\hline $\begin{array}{l}\text { Trastomo digestivo } \\
\text { intenso y transitorio }\end{array}$ & $p$ & $\begin{array}{l}\text { Agaricns .xanthodermus, } \\
\text { Hebeloma crustuliniforme } \\
\text { Hebelomu spp., Tricholoma } \\
\text { yessundatum, Hypholoma spp. } \\
\text { Hypholoma sublateritimm, } \\
\text { Scleroderma antantium! }\end{array}$ & I a 2 hrs. & Gastroenteritis & $6-18$ lirs. \\
\hline \multicolumn{2}{|c|}{$\begin{array}{l}\text { Trastorno digestivo } \\
\text { ligero,ocasional y transitorio }\end{array}$} & $\begin{array}{l}\text { Lepista muda, Armillaria } \\
\text { mellea, Suillus gramularus }\end{array}$ & 1 a 2 lirs. & Gastroenteritis ligera & $8-.12$ hrs. \\
\hline
\end{tabular}

